\title{
ON VARIOUS TYPES OF BARRELLEDNESS AND THE HEREDITARY PROPERTY OF $(D F)$-SPACES
}

\author{
by T. HUSAIN $\dagger$ and YAU-CHUEN WONG
}

(Received 1 April, 1975; revised 20 October, 1975)

1. Introduction. Recently, Levin and Saxon [5], De Wilde and Houet [2] defined the $\sigma$-barrelledness while Husain [3] defined the countable barrelledness and countable quasibarrelledness. It is well-known that barrelled spaces are countably barrelled, and countably barrelled spaces are $\sigma$-barrelled. It is natural to ask whether there is some condition for $\sigma$-barrelled (resp. countably barrelled) spaces to be countably barrelled (resp. barrelled). Using the concept of $S$-absorbent sequences of sets, we are able to give such conditions in Theorem 2.5 and Corollaries 2.6 and 2.7.

Valdivia [9], Saxon and Levin [8] have shown that every vector subspace with countable codimension of a barrelled space is barrelled. Also Levin and Saxon showed in [5] that this hereditary property is true for $\sigma$-barrelled spaces. In $\$ 3$, we show that this hereditary property is also true for countably barrelled spaces as well as for $\sigma$-barrelled $(D F)$-spaces, which is a generalization of Valdivia [10, Theorem 3].

The final section is devoted to some properties of $S$-absorbent sequences of sets which extend some results of Valdivia [9], De Wilde and Houet [2].

2. The relationship between various types of barrelledness. Let $(E, T)$ be a Hausdorff locally convex space whose topological dual is denoted by $E^{\prime}$. If $B$ is a subset of $E$ (resp. $E^{\prime}$ ), then the polar of $B$, taken in $E^{\prime}$ (resp. $E$ ), is denoted by $B^{0}$. By a topologizing family (t. family, for short) for $E^{\prime}$ (resp. $E$ ) we mean a family $S$ consisting of (convex circled) $\sigma\left(E, E^{\prime}\right.$ )-bounded subsets of $E$ (resp. $\sigma\left(E^{\prime}, E\right.$ )-bounded subsets of $E^{\prime}$ ) such that $\cup\{B: B \in S\}=E$ (resp. $E^{\prime}$ ). For a t. family $S$ for $E^{\prime}$ (resp. $E$ ), the topology on $E^{\prime}$ (resp. $E$ ) of uniform convergence on $S$ is denoted by $T_{S}$.

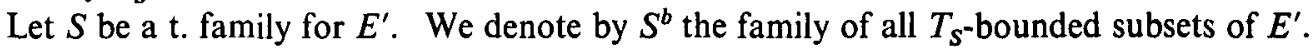
Clearly $S^{b}$ is again a $t$. family for $E$. The topology on $E$ of uniform convergence on $S^{b}$ is denoted by $T_{S}^{b}$, therefore we have $T_{S}^{b}=T_{S^{b}}$. Similarly we can define $S^{n b}$ and $T_{S}^{n b}$, where $S^{n b}=S^{b b \ldots b}$ and $T_{S}^{n b}=T_{S}^{h b \ldots b}$, the superscript $b$ being repeated $n$ times in each case; consequently we have $T_{S}^{n b}=T_{S^{n b}}$ for all $n \geqq 1$.

If $S$ is a t. family for $E^{\prime}$, let us say temporarily that $S^{b}$ (resp. $S^{b b}$ ) is the bounded-polar (resp. bounded-bipolar) family of $S$, and that $T_{S}^{b}$ (resp. $T_{S}^{b b}$ ) is the bounded-polar (resp. boundedbipolar) topology of $T_{S}$. It is clear that $\left\{S^{0}: S \in S^{b}\right\}$ forms a neighbourhood base at 0 for the bounded-polar topology $T_{S}^{b}$, and that $\left\{B^{0}: B \in S^{b b}\right\}$ forms a neighbourhood base at 0 for the bounded-bipolar topology $T_{S}^{b b}$. If $S_{1}$ and $S_{2}$ are two t. families for $E^{\prime}$ with $S_{1} \subset S_{2}$, then $S_{2}^{b} \subset S_{1}^{b}$.

LEMMA 2.1. For a $t$. family $S$ for $E^{\prime}$, we have:

(a) $S \subset S^{b b}$;

† This research was supported by an N.R.C. grant. 
(b) $T_{s} \leqq T_{s}^{b b}$;

(c) $S^{b}=S^{3 b}$ and $T_{S}^{b}=T_{S}^{3 b}$.

The proof is straightforward and will be omitted. We shall see that the inclusion in (a) may be strict.

In the sequel we denote by $\beta\left(E, E^{\prime}\right)$ the strong topology on $E$, i.e., the topology of uniform convergence on all $\sigma\left(E^{\prime}, E\right)$-bounded subsets of $E^{\prime}$, and by $\beta^{*}\left(E, E^{\prime}\right)$ the topology of uniform convergence on all $\beta\left(E^{\prime}, E\right)$-bounded subsets of $E^{\prime}$. As usual, $\tau\left(E, E^{\prime}\right)$ denotes the Mackey topology on $E$. Clearly,

$$
\tau\left(E, E^{\prime}\right) \leqq \beta^{*}\left(E, E^{\prime}\right) \leqq \beta\left(E, E^{\prime}\right)
$$

and

$$
\tau\left(E^{\prime}, E\right) \leqq \beta^{*}\left(E^{\prime}, E\right) \leqq \beta\left(E^{\prime}, E\right) .
$$

It is not hard to see that each $\sigma\left(E, E^{\prime}\right)$-bounded subset of $E$ is $\beta^{*}\left(E, E^{\prime}\right)$-bounded, and that dually each $\sigma\left(E^{\prime}, E\right)$-bounded subset of $E^{\prime}$ is $\beta^{*}\left(E^{\prime}, E\right)$-bounded.

EXAMPLes. (1) If $S_{f}$ is the family of all finite subsets of $E$, then we have that $T_{S_{f}}=\sigma\left(E^{\prime}, E\right) ; \quad T_{S_{f}}^{b}=\beta\left(E, E^{\prime}\right) ; T_{S_{f}}^{b b}=\beta^{*}\left(E^{\prime}, E\right)$. Therefore we conclude that $S_{f} \neq S_{f}^{b b}$ and $T_{S_{f}} \neq T_{S_{f}}^{b b}$ in general.

(2) If $S_{\beta}$ is the family of all $\beta\left(E, E^{\prime}\right)$-bounded subsets of $E$, then we have $T_{s_{\beta}}=\beta^{*}\left(E^{\prime}, E\right)$, $S_{\beta}^{h}$ is the family of all $\sigma\left(E^{\prime}, E\right)$-bounded subsets of $E^{\prime}$ and $T_{S_{\beta}}^{b}=\beta\left(E, E^{\prime}\right)$. Therefore we conclude that $S_{\beta}=S_{\beta}^{b b}$ and $T_{S_{\beta}}=T_{s_{\beta}}^{b b}$.

(3) If $S_{\sigma}$ is the family of all $\sigma\left(E, E^{\prime}\right)$-bounded subsets of $E$, then we have $T_{S_{\sigma}}=\beta\left(E^{\prime}, E\right)$, $T_{S_{\sigma}}^{b}=\beta^{*}\left(E, E^{\prime}\right)$ and $S_{\sigma}^{b b}$ is the family of all $\sigma\left(E, E^{\prime}\right)$-bounded subsets of $E$.

(4) Let $S_{c}$ be the family of all $T$-compact convex circled subsets of $E$ and let $c\left(E^{\prime}, E\right)$ be the topology on $E^{\prime}$ of uniform convergence on $S_{c}$. Then $\sigma\left(E^{\prime}, E\right) \leqq c\left(E^{\prime}, E\right) \leqq \tau\left(E^{\prime}, E\right)$; furthermore we have $T_{S_{c}}=c\left(E^{\prime}, E\right), T_{S_{c}}^{b}=\beta\left(E, E^{\prime}\right)$ and $T_{S_{c}}^{b b}=\beta^{*}\left(E^{\prime}, E\right)$.

Definition 2.2. Let $(E, T)$ be a locally convex space and $S$ a t. family for $E^{\prime}$. Then $E$ is said to be

(1) $S$-barrelled if each member in $S^{b}$ is $T$-equicontinuous;

(2) countably $S$-barrelled if each member of $S^{b}$ which is the countable union of $T$ equicontinuous subsets of $E$ is $T$-equicontinuous;

(3) $\sigma-S$-barrelled if each member in $S^{b}$ which is a countable set is $T$-equicontinuous.

If $S$ is the family of all finite subsets of $E$, then $E$ is $S$-barrelled (resp. countably $S$-barrelled, $\sigma$-S-barrelled) if and only if it is barrelled (resp. countably barrelled, $\sigma$-barrelled) under the usual terminology of [4] and [6] (resp. [3], [2]). $\sigma$-barrelled spaces are also called $\omega$-barrelled by Levin and Saxon [5]. Clearly each $\beta\left(E^{\prime}, E\right)$-bounded set is in $S^{b}$ for any t. family for $E^{\prime}$. Hence $E$ is quasibarrelled (or countably quasibarrelled or $\sigma$-evaluable) if $E$ is $S$-barrelled (or countably $S$-barrelled or $\sigma-S$-barrelled).

If $S$ is the family of all $\sigma\left(E, E^{\prime}\right)$-bounded subsets of $E$, then $E$ is $S$-barrelled (resp. countably $S$-barrelled, $\sigma-S$-barrelled) if and only if $E$ is quasibarrelled (resp. countably 
quasibarrelled, $\sigma$-evaluable) under the usual terminology of [4] [6] (resp. [3], [2]). Here we call $\sigma$-evaluable spaces $\sigma$-infrabarrelled.

If $(E, T)$ is a locally convex Riesz space and if $S$ is the family of all order-bounded subsets of $E$, then $E$ is $S$-barrelled if and only if it is order-infrabarrelled under the usual definition of [11].

As a consequence of Lemma 2.1, we have the following result.

LEMma 2.3. Let $S$ be a t. family for $E^{\prime} . \quad E$ is $S$-barrelled (resp. countably $S$-barrelled, $\sigma$-S-barrelled) if and only if $E$ is $S^{b b}$-barrelled (resp. countably $S^{b b}$-barrelled, $\sigma-S^{b b}$-barrelled).

In particular, $(E, T)$ is barrelled if and only if each $\sigma\left(E, E^{\prime}\right)$-closed convex circled subset of $E$ which absorbs all $\beta\left(E, E^{\prime}\right)$-bounded subsets of $E$ is a $T$-neighbourhood of 0 .

Using a standard argument, for instance, see Schaefer [6] and Köthe [4, p. 396], it is easily seen that $E$ is $S$-barrelled if and only if each closed convex circled subset of $E$ which absorbs all members of $S$ is a $T$-neighbourhood of 0 , and that $E$ is countably $S$-barrelled if and only if for any sequence $\left(V_{n}\right)$ of closed convex circled $T$-neighbourhoods of 0 , if $V=\bigcap_{n=1}^{\infty} V_{n}$
absorbs all members in $S$ then $V$ is a $T$-neighbourhood of 0 .

In order to give a dual characterization of the $\sigma$-S-barrelledness, we require the following terminology. Let $S$ be a t. family for $E^{\prime}$. By an $S$-absorbent sequence (of closed sets) in $E$ we mean a sequence $\left\{V_{n}: n \geqq 1\right\}$ of (closed) convex circled sets in $E$ for which the following two conditions are satisfied:

(i) $V_{n} \subset V_{n+1}$ for all $n \geqq 1$;

(ii) each member in $S$ is absorbed by some $V_{n}$.

If $S$ is the family of all finite subsets of $E$, then $\left\{V_{n}: n \geqq 1\right\}$ is an $S$-absorbent sequence if and only if it is an absorbent sequence in $E$ in the sense of [2]; and if $S$ is the family of all $\sigma\left(E, E^{\prime}\right)$-bounded subsets of $E$, then $\left\{V_{n}: n \geqq 1\right\}$ is a $\sigma$-absorbent sequence if and only if it is a bounded-absorbent sequence in the sense of [2].

Proposition 2.4. Let $S$ be a $t$. family for $E^{\prime}$. Then $E$ is $\sigma$-S-barrelled if and only if for any $S$-absorbent sequence $\left\{V_{n}: n \geqq 1\right\}$ in $E$, the sequence $\left\{f_{n}: n \geqq 1\right\}$ is equicontinuous, where $f_{n} \in V_{n}^{0}$ for all $n \geqq 1$.

Proof. Necessity. For any $S \in S$ there exists $\lambda>0$ and $n_{0}>1$ such that $S \subset \lambda V_{n}$ for all $n \geqq n_{0}$. For each $n \geqq 1$, let $f_{n} \in V_{n}^{0}$. Then $\left|f_{n}(x)\right| \leqq \lambda$ for all $x \in S$ and $n \geqq n_{0}$. Since $S$ is $\sigma\left(E, E^{\prime}\right)$-bounded, there exists $\mu>0$ with $\left|f_{n}(x)\right| \leqq \mu$ for all $x \in S$ and $n=1, \ldots, n_{0}-1$. Thus $\sup \left\{\left|f_{n}(x)\right|: x \in S, n \geqq 1\right\} \leqq \max (\lambda, \mu)<\infty$ and so $\left\{f_{n}: n \geqq 1\right\} \in S^{b}$. Hence by hypothesis $\left\{f_{n}: n \geqq 1\right\}$ is equicontinuous.

Sufficiency. Let $\left\{h_{n}: n \geqq 1\right\}$ be a $T_{s}$-bounded sequence in $E^{\prime}$. For each $k \geqq 1$, we define

$$
V_{k}=\left\{x \in E:\left|h_{n}(x)\right| \leqq 1 \text { for all } n \geqq k\right\} \text {. }
$$

Then $\left\{V_{n}: n \geqq 1\right\}$ is an $S$-absorbent sequence in $E$. As $h_{k} \in V_{k}^{0}$ for all $k \geqq 1$, we conclude from the hypothesis that $\left\{h_{k}: k \geqq 1\right\}$ is equicontinuous. This shows that $E$ is $\sigma$-S-barrelled. 
If $S_{1}$ and $S_{2}$ are two t. families for $E^{\prime}$ such that $S_{1} \subset S_{2}$, then the following implications hold:

$$
\begin{gathered}
S_{1} \text {-barrelledness } \Rightarrow \text { countably } S_{1} \text {-barrelledness } \Rightarrow \sigma-S_{1} \text {-barrelledness } \\
\Downarrow \\
\Downarrow \\
S_{2} \text {-barrelledness } \Rightarrow \text { countably } S_{2} \text {-barrelledness } \Rightarrow \sigma-S_{2} \text {-barrelledness. }
\end{gathered}
$$

Therefore it is natural to ask under what conditions on $E$ (or $E^{\prime}$ ) the corresponding converse implications hold. We have the following result.

THEOREM 2.5. Let $S_{1}$ and $S_{2}$ be two $t$. families for $E^{\prime}$ such that $S_{1} \subset S_{2}$. Then $(E, T)$ is $\sigma-S_{1}$-barrelled (resp. countably $S_{1}$-barrelled, $S_{1}$-barrelled) if and only if the following two conditions hold:

(i) $E$ is $\sigma-S_{2}$-barrelled (resp. countably $S_{2}$-barrelled, $S_{2}$-barrelled);

(ii) each $S_{1}$-absorbent sequence of closed sets in $E$ is $S_{2}$-absorbent.

Proof. Suppose that $E$ is $\sigma-S_{1}$-barrelled and that $\left\{V_{n}: n \geqq 1\right\}$ is an $S_{1}$-absorbent sequence of closed sets in $E$ which is not $S_{2}$-absorbent. Then there exists $B \in S_{2}$ such that $B \subset n V_{n}$ is false for all natural numbers $n \geqq 1$. For each $n \geqq 1$, let $x_{n}$, in $B$, be such that $x_{n} \notin n V_{n}$. As $V_{n}$ is closed convex and circled, the bipolar theorem ensures that there exists $f_{n} \in V_{n}^{0}$ such that

$$
\left|f_{n}\left(x_{n}\right)\right|>n \text {. }
$$

As $E$ is $\sigma$ - $S_{1}$-barrelled and $\left\{V_{n}: n \geqq 1\right\}$ is an $S_{1}$-absorbent sequence of closed sets in $E$, it follows from Proposition 2.4 that $\left\{f_{n}: n \geqq 1\right\}$ is a $T$-equicontinuous sequence, and hence that $\left\{f_{n}: n \geqq 1\right\}$ is $T_{S_{2}}$-bounded; consequently $\left\{f_{n}: n \geqq 1\right\}$ must be absorbed by $B^{0}$, contrary to the inequality (1). Therefore the conditions are necessary. We show that the conditions are also sufficient.

Let $\left\{f_{n}: n \geqq 1\right\}$ be a $T_{S_{1}}$-bounded sequence in $E^{\prime}$. For each $k \geqq 1$, let

$$
V_{k}=\left\{x \in E:\left|f_{n}(x)\right| \leqq 1 \text { for all } n \geqq k\right\} \text {. }
$$

The $T_{S_{1}}$-boundedness of $\left\{f_{n}: n \geqq 1\right\}$ ensures that $\left\{V_{n}: n \geqq 1\right\}$ is an $S_{1}$-absorbent sequence of closed sets in $E$, and hence $\left\{V_{n}: n \geqq 1\right\}$ is $S_{2}$-absorbent by the hypotheses. On the other hand, since $E$ is assumed to be $\sigma-S_{2}$-barrelled and since $f_{n} \in V_{n}^{0}$ for all $n \geqq 1$, it follows from Proposition 2.4 that $\left\{f_{n}: n \geqq 1\right\}$ is $T$-equicontinuous, and hence that $E$ is $\sigma-S_{1}$-barrelled.

The necessity part of the proof for countably $S_{1}$-barrelled and $S_{1}$-barrelled spaces is similar and so is omitted. The sufficiency part for all cases can be handled as follows. Observe that $S_{1}^{b} \supset S_{2}^{b}$. To show that (ii) implies $S_{1}^{b}=S_{2}^{b}$, let $A \in S_{1}^{b}$ and $A \notin S_{2}^{b}$. Then there is $B \in S_{2}$, a sequence $\left\{x_{n}\right\} \subset B$ and a sequence $\left\{f_{n}\right\} \subset A$ such that $\left|f_{n}\left(x_{n}\right)\right|>n$ for all $n \geqq 1$. Since $V_{n}=\left\{x \in E:\left|f_{m}(x)\right| \leqq 1\right.$ for $\left.m \geqq n\right\}$ is an $S_{1}$-absorbent sequence of closed sets in $E$, it follows by (ii) that it is also $S_{2}$-absorbent. Hence there exist $n$ and $\lambda$ such that $B \subset \lambda V_{n}$, a contradiction.

REMARK. $E$ is $\sigma$-barrelled (resp. countably barrelled, barrelled) if and only if it is $\sigma$-infrabarrelled (resp. countably quasibarrelled, infrabarrelled) and each absorbent sequence of closed sets in $E$ is bounded-absorbent. 
Corollary 2.6. Let $S_{1}$ and $S_{2}$ be two t. families for $E^{\prime}$ such that $S_{1} \subset S_{2}$. Then:

(a) $E$ is countably $S_{1}$-barrelled if and only if it is countably $S_{2}$-barrelled as well as $\sigma-S_{1}$-barrelled;

(b) $E$ is $S_{1}$-barrelled if and only if it is $\sigma-S_{1}$-barrelled as well as $S_{2}$-barrelled.

Proof. If $E$ is countably $S_{1}$-barrelled, then it is obvious that $E$ is countably $S_{2}$-barrelled as well as $\sigma-S_{1}$-barrelled. Conversely, if $E$ is countably $S_{2}$-barrelled and if $E$ is $\sigma-S_{1}$-barrelled, then by Theorem 2.5 , each $S_{1}$-absorbent sequence of closed sets in $E$ is $S_{2}$-absorbent. We conclude from Theorem 2.5 again that $E$ is countably $S_{1}$-barrelled. This proves the assertion (a). The proof of (b) is similar.

REMARK. $E$ is countably barrelled if and only if it is $\sigma$-barrelled and countably quasibarrelled $E$ is barrelled if and only if it is countably barrelled and quasibarrelled.

Corollary 2.7. Let $S_{1}$ and $S_{2}$ be two $t$. families for $E^{\prime}$ such that $S_{1} \subset S_{2}$. Then the following assertions hold.

(a) Let $E$ be countably $S_{2}$-barrelled. Then $E$ is countably $S_{1}$-barrelled if and only if each $S_{1}$-absorbent sequence of closed sets in $E$ is $S_{2}$-absorbent.

(b) Let $E$ be $S_{2}$-barrelled. Then $E$ is $S_{1}$-barrelled if and only if $E$ is $\sigma-S_{1}$-barrelled, and this is the case if and only if each $S_{1}$-absorbent sequence of closed sets in $E$ is $S_{2}$-absorbent.

Proof. (a) follows from Theorem 2.5 and Corollary 2.6 (a), while (b) follows from Corollary 2.6 (b) and the assertion (a) of this corollary.

Let $E$ be a locally convex space. A convex circled $\sigma\left(E, E^{\prime}\right)$-bounded subset $B$ of $E$ is said to be infracomplete if the normed space $E(B)=\bigcup_{n} n B$ equipped with the norm $\|\cdot\|_{B}$ defined by

$$
\|x\|_{B}=\inf \{\lambda \geqq 0: x \in \lambda B\} \quad(x \in E(B))
$$

is complete. It is clear that every convex circled $\sigma\left(E, E^{\prime}\right)$-bounded and $\tau\left(E, E^{\prime}\right)$-sequentially complete subset of $E$ is infracomplete. By the Banach-Mackey theorem, we see that every infracomplete subset $B$ of $E$ is $\beta\left(E, E^{\prime}\right)$-bounded (see [4, $\left.\$ 20,11(3)\right]$ ).

Levin and Saxon [5] say that a locally convex space $E$ has the property $(C)$ (resp. the property $(S)$ ) if every $\sigma\left(E^{\prime}, E\right)$-bounded subset of $E^{\prime}$ is $\sigma\left(E^{\prime}, E\right)$-relatively countably compact (resp. $E^{\prime}$ is $\sigma\left(E^{\prime}, E\right)$-sequentially complete). As a consequence of the result mentioned above $([4, \S 20,11(3)])$, we obtain the following result which gives a connection between $\sigma$-barrelledness and the property $(S)$.

Proposition 2.8. For a $\sigma$-infrabarrelled locally convex space $E$, the following statements are equivalent:

(a) $E$ is $\sigma$-barrelled;

(b) $E$ has the property $(C)$;

(c) $E$ has the property $(S)$;

(d) each $\sigma\left(E^{\prime}, E\right)$-bounded, $\sigma\left(E^{\prime}, E\right)$-closed subset of $E^{\prime}$ is $\sigma\left(E^{\prime}, E\right)$-sequentially complete. 
Proof. The implications (a) $\Rightarrow$ (b) $\Rightarrow$ (c) $\Rightarrow$ (d) are obvious. Finally, if the statement (d) holds, then by the Banach-Mackey theorem each $\sigma\left(E^{\prime}, E\right)$-bounded subset of $E^{\prime}$ is $\beta\left(E^{\prime}, E\right)$ bounded, and thus the implication (d) $\Rightarrow$ (a) follows.

Consider the vector space $m$ of all bounded sequences with the Mackey topology $\tau\left(m, l_{1}\right)$. Levin and Saxon have shown in [5, Proposition 6] that $\left(m, \tau\left(m, l_{1}\right)\right)$ is a Mackey space with the property $(S)$ but not property $(C)$. According to this result and Proposition 2.8, we conclude that Mackey spaces are, in general, not $\sigma$-infrabarrelled spaces.

As another consequence of the Banach-Mackey theorem, we have the following result.

Proposition 2.9. Let $E$ be a locally convex space for which every $\sigma\left(E^{\prime}, E\right)$-bounded closed set is $\sigma\left(E^{\prime}, E\right)$-sequentially complete (equivalently, $E$ has the property $(S)$ ). Then the following assertions hold.

(1) If $E$ is infrabarrelled (in particular, bornological) then it is barrelled.

(2) If $E$ is countably infrabarrelled then it is countably barrelled.

Proof. According to the Banach-Mackey theorem each $\sigma\left(E^{\prime}, E\right)$-bounded subset of $E^{\prime}$ is $\beta\left(E^{\prime}, E\right)$-bounded, and the result follows.

Since metrizable locally convex spaces are infrabarrelled, part (1) of the preceding result is a generalization of Saxon [7, Theorem 2.7]. The following corollary is now immediate.

COROLLARY 2.10. Let $E$ be a locally convex space in which every $\sigma\left(E, E^{\prime}\right)$-bounded closed set is $\tau\left(E, E^{\prime}\right)$-sequentially complete (in particular, $E$ is either $\tau\left(E, E^{\prime}\right)$-sequentially complete or quasi-complete). Then the following assertions hold.

(1) If $E$ is $\sigma$-infrabarrelled then $E$ is $\sigma$-barrelled and a fortiori has the property $(S)$.

(2) If $E$ is countably infrabarrelled (resp. barrelled) then it is countably barrelled (resp. barrelled).

3. The hereditary property. Saxon, Levin [8] and Valdivia [9] have shown independently that a vector subspace with countable codimension of a barrelled space is barrelled. Also Saxon and Levin [5] have shown that a vector subspace with countable codimension of a $\sigma$-barrelled space is $\sigma$-barrelled. The same is true for countably barrelled spaces as shown by Webb [12]. We give a different and direct proof of this fact.

THEOREM 3.1. Let $M$ be a countable codimensional vector subspace of a countably barrelled space $E$. Then $M$ is countably barrelled when furnished with the relative topology.

Proof. In our proof we consider three cases.

(a) $M$ is dense in $E$. In this case, the topological dual $M^{\prime}$ of $M$ can be canonically identified with $E^{\prime}$. Let $S$ be a $\sigma\left(M^{\prime}, M\right)$-bounded subset of $M^{\prime}$ and let $\left\{S_{n}: n \geqq 1\right\}$ be a sequence of equicontinuous subsets of $M^{\prime}$ for which $S=\bigcup_{n=1}^{\infty} S_{n}$. Since $M$ is dense in $E$, it follows from [5, Lemma 2] that $S$ is $\sigma\left(E^{\prime}, E\right)$-bounded. Further we show that each $S_{n}$ is an equicontinuous subset of $E^{\prime}$. 
In fact, let $S_{n}^{0}$ denote the polar of $S_{n}$ taken in $E$. Since $S_{n}$ is an equicontinuous subset of $M^{\prime}, S_{n}^{0} \cap M$ is a 0-neighbourhood in $M$; then there exists an open 0-neighbourhood $U_{n}$ in $E$ such that $U_{n} \cap M \subset S_{n}^{0} \cap M \subset S_{n}^{0}$. The density of $M$ ensures that $U_{n} \subset \overline{U_{n} \cap M} \subset S_{n}^{0}$, and hence $S_{n}$ is an equicontinuous subset of $E^{\prime}$.

Now the countable barrelledness of $E$ implies that $S$ is an equicontinuous subset of $E^{\prime}$ and surely an equicontinuous subset of $M^{\prime}$. This shows that $M$ is countably barrelled.

(b) $M$ is closed in $E$. Let $N$ be any algebraic complement to $M$ in $E$. Since countably barrelled spaces are $\sigma$-barrelled, it follows from [7, Theorem 1.1] that $N$ is a topological complement and has the strongest locally convex topology. Hence $N$ is closed in $E, M$ and $E / N$ are topologically isomorphic. Since $E$ is countably barrelled, by [3, Corollary 14], $E / N$ is countably barrelled and therefore $M$ must be countably barrelled.

(c) General case. Since $\bar{M}$ is a closed vector subspace of $E$ with countable codimension, it follows from (b) that $\bar{M}$ is countably barrelled. As $M$ is dense in $\bar{M}$, we conclude from (a) that $M$ is countably barrelled. This completes the proof of the theorem.

COROLlary 3.2. Let $E$ be a $\sigma$-barrelled (DF)-space. Then any vector subspace $M$ of $E$ with countable codimension is a countably barrelled $(D F)$-space.

Proof. By Corollary 2.6, $E$ is a countably barrelled $(D F)$-space, and hence $M$ is a countably barrelled space by the preceding theorem. Since $E$ has a countable fundamental system of bounded sets, and since $M$ is a subspace, it follows that $M$ contains a countable fundamental system of bounded subsets of $M$. Therefore $M$ is a countably barrelled $(D F)$-space.

The preceding result was proved by Valdivia [10, Theorem 3] in the special case when $E$ is barrelled.

4. Various types of absorbent sequences. Let $E$ be a vector space. By an increasing sequence of sets in $E$ we mean a sequence $\left\{V_{n}: n \geqq 1\right\}$ of convex circled subsets of $E$ such that $V_{n} \subset V_{n+1}$ for all $n \geqq 1$. Let $\left\{V_{n}: n \geqq 1\right\}$ be an increasing sequence of sets in $E$. It is clear that $\left\{n V_{n}: n \geqq 1\right\}$ is an increasing sequence of sets in $E$, and that if $E$ is a locally convex space then $\left\{\bar{V}_{n}: n \geqq 1\right\}$ is also an increasing sequence of sets in $E$, where $\bar{V}_{n}$ is the closure of $V_{n}$. An increasing sequence $\left\{V_{n}: n \geqq 1\right\}$ of sets in $E$ is called an increasing sequence of $(P)$ sets in $E$ if each $V_{n}$ has the property $(P)$; for instance, $\left\{V_{n}: n \geqq 1\right\}$ is an increasing sequence of closed (resp. complete, compact, metrizable etc.) sets in $E$ if each $V_{n}$ is closed (resp. complete, compact, metrizable etc.).

It is known from $\$ 2$ that the concept of $S$-absorbent sequences is useful for studying the relationship between various types of barrelledness. It is not hard to give an example of an increasing sequence of sets in $E$ which is not $S$-absorbent. Therefore it is interesting to find some sufficient and necessary condition to ensure that increasing sequences are $S$-absorbent.

Proposition 4.1. Let $S$ be a $t$. family for $E^{\prime}$ and suppose that $\left\{V_{n}: n \geqq 1\right\}$ is an increasing sequence of closed sets in $E$. Then it is an $S$-absorbent sequence if and only if for any $f_{n} \in V_{n}^{0}(n \geqq 1)$, the sequence $\left\{f_{n}: n \geqq 1\right\}$ is $T_{s}$-bounded. 
Proof. Suppose that $\left\{V_{n}: n \geqq 1\right\}$ is $S$-absorbent and that $\left\{f_{n}: n \geqq 1\right\}$ is not $T_{s}$-bounded for some $f_{n} \in V_{n}^{0}(n \geqq 1)$. Then there exists $B \in S$ such that $\left\{f_{n}: n \geqq 1\right\} \subset k^{2} B^{0}$ is false for all natural numbers $k \geqq 1$. For each $k \geqq 1$, there exists $n_{k}$ such that $f_{n_{k}} \notin k^{2} B^{0}$. On the other hand, since $\left\{V_{n}: n \geqq 1\right\}$ is $S$-absorbent, there exists $\lambda>0$ and $n_{0} \geqq 1$ such that

$$
V_{n}^{0} \subset V_{n_{0}}^{0} \subset \lambda B^{0} \text { for all } n \geqq n_{0},
$$

it then follows that $f_{n} \in \lambda B^{0}$ for all $n \geqq n_{0}$, which contradicts the fact that $f_{n_{k}} \notin k^{2} B^{0}$. Therefore the condition is necessary.

Conversely, if $\left\{V_{n}: n \geqq 1\right\}$ is not an $S$-absorbent sequence, then there exists $B \in S$ such that $B \subset n V_{n}$ is false for all natural numbers $n \geqq 1$. For each $n$, let $x_{n} \in B \backslash\left(n V_{n}\right)$ and let $f_{n}$, in $V_{n}^{0}$, be such that $\left|f_{n}\left(x_{n}\right)\right|>n$. Then the sequence $\left\{f_{n}: n \geqq 1\right\}$ is not $T_{s}$-bounded. This completes the proof.

In the sequel we always assume that $E$ is a locally convex space and that $S$ is a topologizing family for $E^{\prime}$. If $S_{1}$ is another topologizing family for $E^{\prime}$ such that $S \subset S_{1}$, then each $S_{1}$-absorbent sequence in $E$ must be $S$-absorbent. The converse is true for $S_{1}=S^{b b}$ as the following result shows.

COROLLARY 4.2. $\left\{V_{n}: n \geqq 1\right\}$ is an $S$-absorbent sequence of closed sets in $E$ if and only if it is an $S^{b b}$-absorbent sequence.

Proof. This follows from Proposition 4.1 and Lemma 2.1.

The preceding result was proved by De Wilde and Houet [2, Theorem 1] in the case when $S$ is the family of all finite subsets of $E$.

Corollary 4.3. Let $S_{1}$ and $S_{2}$ be two $t$. families for $E^{\prime}$ such that $S_{1} \subset S_{2}$. Then the following statements are equivalent:

(i) each $T_{S_{1}}$-bounded subset of $E^{\prime}$ is $T_{S_{2}}$-bounded;

(ii) each $S_{1}$-absorbent sequence of closed sets in $E$ is $S_{2}$-absorbent.

Proof. The implication (i) $\Rightarrow$ (ii) follows from Proposition 4.1, while the implication (ii) $\Rightarrow$ (i) has been observed in Theorem 2.5 .

When $S_{1}$ is the family of all finite subsets of $E$ and $S_{2}$ is the family of all $\sigma\left(E, E^{\prime}\right)$-bounded subsets of $E$, then the implication (i) $\Rightarrow$ (ii) in the preceding result was proved by Valdivia [9, Theorem 6] in the case when $E$ is barrelled, and was proved by De Wilde and Houet [2, Corollary 1] in the case when $E$ is $\sigma$-barrelled.

By making use of Theorem 2.5, for a $\sigma$-barrelled space $E$, each $\sigma\left(E^{\prime}, E\right)$-bounded subset of $E^{\prime}$ is $\beta\left(E^{\prime}, E\right)$-bounded.

Corollary 4.4. Let $S_{1}$ and $S_{2}$ be two $t$. families for $E^{\prime}$ such that $S_{1} \subset S_{2}$, and let $E$ satisfy one of the equivalent conditions (i) and (ii) of Corollary 4.3. If $S_{2}$ has a sequence $\left\{B_{n}: n \geqq 1\right\}$ such that each member of $S_{1}$ is absorbed by some $B_{n}$, then the saturated hull ([6], p. 81) of $S_{2}$ contains a countable fundamental subfamily.

Proof. For each $n$, let $V_{n}$ be the closed convex circled hull of $\bigcup_{j=1}^{n} B_{j}$. Then $V_{n}$ is in the saturated hull of $S_{2}$, and $\left\{V_{n}: n \geqq 1\right\}$ is an $S_{1}$-absorbent sequence of closed sets in $E$, so by 
the hypothesis, $\left\{V_{n}: n \geqq 1\right\}$ is $S_{2}$-absorbent. Consequently $\left\{n V_{n}: n \geqq 1\right\}$ is a countable fundamental subfamily of the saturated hull of $S_{2}$ because a member of the saturated hull of $S_{2}$ is either a subset, scalar multiple or an absolute convex hull of a finite number of elements of $S_{2}$.

REMARK. If $E$ is a countably barrelled space with a sequence $\left\{B_{n}: n \geqq 1\right\}$ of bounded sets such that $\bigcup_{n=1}^{\infty} B_{n}$ is absorbing, then $E$ is a $(D F)$-space.

Corollary 4.4 was proved by Valdivia [9, Corollary 2.6] in the case when $E$ is barrelled.

A trivial modification of De Wilde and Houet's argument in [2] yields the following more general result, but for completeness we shall give the entire proof.

THEOREM 4.5. Let $E$ be a $\sigma$-S-barrelled space and $\left\{V_{n}: n \geqq 1\right\}$ an S-absorbent sequence in $E$. Then

$$
\overline{\bigcup_{m}^{\infty} V_{m}} \subset(1+\varepsilon) \bigcup_{m}^{\infty} \bar{V}_{m} \text { for all } \varepsilon>0 .
$$

Proof. If $x \notin(1+\varepsilon) \bigcup_{m}^{\infty} \bar{V}_{m}$ for some $\varepsilon>0$, then $x \notin(1+\varepsilon) \bar{V}_{m}$ for all $m \geqq 1$, and thus, for any $m \geqq 1$, there exists $f_{m} \in V_{m}^{0}$ such that $f_{m}(x)>1+\varepsilon$. Since $E$ is $\sigma$-S-barrelled, by Proposition $2.4,\left\{f_{m}: m \geqq 1\right\}$ has a $\sigma\left(E^{\prime}, E\right)$-cluster point $f$, say, in $E^{\prime}$; hence $f(x) \geqq 1+\varepsilon$. On the other hand, since $V_{n}$ is increasing and $f_{n} \in V_{n}^{0}$, it follows that $f \in V_{n}^{0}$ for all $n \geqq \frac{1 \text { or, }}{\infty}$ equivalently $f \in \bigcap_{n \geqq 1} V_{n}^{0}=\left(\bigcup_{n \geqq 1} V_{n}\right)^{0}$. However the inequality $f(x) \geqq 1+\varepsilon$ shows that $x \notin \bigcup_{m}^{\infty} V_{m}$. This completes the proof.

Remarks. (1) As De Wilde in [1, p. 212] pointed out, the condition in Theorem 4.5 that $E$ be $\sigma$-S-barrelled can be replaced by the following condition: $\left\{V_{n}: n \geqq 1\right\}$ is an $S$-absorbent sequence in $E$ such that for each $f_{n} \in V_{n}^{0}(n \geqq 1)$, the sequence $\left\{f_{n}: n \geqq 1\right\}$ is equicontinuous.

(2) According to the preceding theorem, Corollaries 2.a-2.d in [2] hold for a $\sigma-S$-barrelled space.

ACKNOWLEDGEMENT. The authors are grateful to the referee for many helpful comments.

\section{REFERENCES}

1. M. De Wilde, Summer school on topological vector spaces, Lecture Notes in Mathematics, No. 331 (Springer-Verlag, 1973).

2. M. De Wilde and C. Houet, On increasing sequences of absolutely convex sets in locally convex spaces, Math. Ann. 192 (1971), 257-261.

3. T. Husain, Two new classes of locally convex spaces, Math. Ann. 166 (1966), 289-299.

4. G. Köthe, Topological vector spaces I (Springer-Verlag, 1969).

5. M. Levin and S. Saxon, A note on the inheritance of properties of locally convex spaces by subspaces of countable codimension, Proc. Amer. Math. Soc. 29 (1971), 97-102.

6. H. H. Schaefer, Topological vector spaces (Springer-Verlag, 1971).

7. S. Saxon, Nuclear and product spaces, Baire-like spaces, and the strongest locally convex topology, Math. Ann. 197 (1972), 87-106. 
8. S. Saxon and M. Levin, Every countable-codimensional subspace of a barrelled space is barrelled, Proc. Amer. Math. Soc. 29 (1971), 91-96.

9. M. Valdivia, Absolutely convex sets in barrelled spaces, Ann. Inst. Fourier Grenoble 21 (2) (1971), 3-13.

10. M. Valdivia, On DF-spaces, Math. Ann. 191 (1971), 38-43.

11. Yau-Chuen Wong, Order-infrabarrelled Riesz spaces, Math. Ann. 183 (1969), 17-32.

12. J. H. Webb, Countable-codimensional subspaces of locally convex spaces, Proc. Edinburgh Math. Soc. 18 (1973), 167-172.

Prof. T. Husain

Department of Mathematics

MCMASTER UNIVERSITY

Hamilton, ONTARIO

Canada
Dr. Yau-Chuen Wong

DEPARTMENT OF MATHEMATICS

UNITED COLLEGE

Chinese University of Hong Kong HONG KONG 